\title{
Displacement current measurement of a pentacene metal-insulator-semiconductor device to investigate both quasi-static and dynamic carrier behavior using a combined waveform
}

\author{
Yuya Tanaka ${ }^{\mathrm{a}}$, Yutaka Noguchi ${ }^{\mathrm{a}, \mathrm{b}}$, Michael Kraus ${ }^{\mathrm{c}}$, Wolfgang Brütting ${ }^{\mathrm{c}}$, \\ Hisao Ishii ${ }^{\mathrm{a}, \mathrm{b}, *}$ \\ ${ }^{a}$ Graduate School of Advanced Integration Science, Chiba University, 1-33 Yayoi-cho, \\ Inage-ku, Chiba 263-8522, Japan \\ ${ }^{b}$ Center for Frontier Science, Chiba University, 1-33 Yayoi-cho, Inage-ku, Chiba \\ 263-8522, Japan \\ ${ }^{c}$ Institute of Physics, University of Augsburg, 86135 Augsburg, Germany
}

\begin{abstract}
Displacement current measurements (DCM) were used to investigate transient carrier behavior during the carrier accumulation process in pentacene (Pn) metal-insulator-semiconductor structures $\left[\mathrm{Au} / \mathrm{Pn}(100 \mathrm{~nm}) / \mathrm{SiO}_{2}(100\right.$ $\mathrm{nm}) / \mathrm{p}$-type $\mathrm{Si}$. We propose a modified DCM technique that applies a combined voltage waveform of ramp and plateau parts to observe the transient carrier behavior. This allows to simultaneously detect both the effective capacitance of the device $\left(C_{\text {eff }}\right)$ and its time dependence $\left(d C_{\text {eff }} / d t\right)$. In addition, the long decay time owing to $d C_{\text {eff }} / d t$ during carrier spreading at the $\mathrm{Pn} / \mathrm{SiO}_{2}$ interface agrees well with the inverse of the relaxation frequency, which was estimated by capacitance-frequency measurement. This result demonstrates that DCM is a powerful tool for investigating both the quasi-static and dynamic behavior of carriers in organic devices.
\end{abstract}

Keywords: Displacement current measurement, Impedance spectroscopy, Pentacene, Carrier injection, Charge transport, Organic electronics

\footnotetext{
*Corresponding author. Tel., fax: +81-43-290-3524.

Email address: ishii130@faculty.chiba-u.jp (Hisao Ishii)
} 


\section{Introduction}

Recently, organic field-effect transistors (OFETs) have been extensively studied because of their potential applications in electronics $[1,2,3]$. Since typical organic semiconductors have an extremely small number of thermally excited carriers and are mostly used without any intentional doping, OFETs are driven by accumulated charge carriers injected from the electrodes $[4,5,6,7]$; this is in contrast to inorganic FETs in which an inversion layer based on actively doped semiconductor material is the dominant mechanism [8]. Therefore, studies have focused on the carrier injection properties as well as the behavior of the injected carriers in the device using various methods, e.g., capacitance-voltage $(C-V)$ measurement $[7,9,10]$, electricfield-induced second-harmonic generation $[11,12,13]$, and displacement current measurement (DCM) $[4,5,14,15,16,17,18,19]$.

Among these methods, DCM has the advantage of directly investigating the transient response of charge carriers. The displacement current through a device is measured under a sweep of a triangular wave voltage. This technique was first used to measure the $C$ - $V$ relationship of organic diodes [14, 15]. Then, Egusa et al. applied this technique to investigate the carrier injection properties in model devices of organic light-emitting diodes [16, 17]. Ogawa et al. extended this method to investigate the carrier behavior in OFETs $[4,5]$. In these studies, DCM was used to observe the carrier dynamics by measuring the effective capacitance of the device. However, Ogawa et al. observed a current peak during the carrier injection process which is not observed in conventional $C$ - $V$ measurements. They suggested that the peak was due to the transient behavior of the injected carriers, although the experimental evidence was insufficient [20]. Furthermore, Liang et al. recently demonstrated the estimation of the carrier mobility in a model OFET device by analyzing the time dependence of the current peak in DCM curves [21].

In principle, the observed current in DCM includes both capacitive and transient terms. The current in metal-insulator-semiconductor (MIS) devices with a time dependent voltage stimulus $V(t)$ is expressed by

$$
I=\frac{d Q}{d t}=C_{\mathrm{eff}} \frac{d V}{d t}+V \frac{d C_{\mathrm{eff}}}{d t}
$$

where $C_{\text {eff }}$ is the effective capacitance of the device, $Q$ is the charge, and $V$ is the applied voltage. Thus, the observed current includes information about both the quasi-static and dynamic carrier behavior as shown in Eq. (1). The 
first term, $C_{\mathrm{eff}}(d V / d t)$, originates from the current in the quasi-static state of the device, while the second term, $V\left(d C_{\text {eff }} / d t\right)$, is due to the dynamic response. In this way, the DCM curve includes both the quasi-static and the dynamic response of the device; however, understanding of the latter is still limited. This is mainly because it is not easy to separate the $V\left(d C_{\text {eff }} / d t\right)$ contribution in a conventional DCM experiment.

In this study, we propose a new DCM scanning mode, called combined waveform DCM method, using a triangular wave with a plateau region which allows to successfully separate the quasi-static and dynamic DCM currents. By applying combined waveform DCM to pentacene (Pn) MIS devices, we demonstrate that the current peak in the DCM curve reported by Ogawa et al. actually originates from the $d C / d t$ term owing to the carrier-spreading process in this device. In addition, the decay time of the transient current in the DCM corresponds to the inverse of the relaxation frequency which is obtained from capacitance-frequency $(C-f)$ measurement. The combined waveform DCM allows us to simultaneously collect information about the effective capacitance and the inverse of the relaxation frequency of the organic device. This advantage will be useful when investigating the device physics in organic electronics.

\section{Method}

As mentioned above, the current in the MIS device (Fig. 1a) is expressed by Eq. (1). We briefly explain the basics of a typical DCM curve, assuming that the device is in a quasi-static state (corresponding to conventional $C$ $V$ measurements). Since the organic and insulator layers beneath the top electrode work as capacitor dielectrics without carrier injection, the observed current is governed by the serially combined capacitance of the organic and insulator layers. The current is given by

$$
I=C_{\mathrm{eff}} \frac{d V}{d t}=\frac{C_{\mathrm{org}} C_{\mathrm{ins}}}{C_{\mathrm{org}}+C_{\mathrm{ins}}} \frac{d V}{d t} .
$$

Here, $C_{\text {org }}$ and $C_{\text {ins }}$ are the capacitance of the organic and insulator layers, respectively. As shown in Fig. $1 \mathrm{~b}$ and $\mathrm{c}$, the start voltage of the triangular wave is set as $0 \mathrm{~V}$, and we assume that the MIS device is completely depleted at the $0 \mathrm{~V}$ [Fig. 1c region 1 and 2]. When holes are injected from the top electrode to the organic layer at the negative voltage [Fig. 1c region 3], they accumulate at the organic/insulator interface as shown in Fig. 1a. Assuming 
that the current flows through the organic layer without a significant voltage drop, only the insulator layer works as a capacitor dielectric, and hence the displacement current can be expressed as

$$
I=C_{\mathrm{ins}} \frac{d V}{d t} .
$$

Since the capacitance of the insulator layer is larger than the combined capacitance, the current increases owing to a hole injection in the backward scan $(d V / d t<0)$.

At the beginning of the forward voltage scan $(d V / d t>0)$, the holes are discharged from the organic layer to the electrode [Fig. 1c region 4]; then, the organic layer is depleted and back to its initial state. As a result, $I-V$ curves of the typical MIS structure are obtained, as shown in Fig. 1c, when the dynamic current is negligibly small.

Conventional DCM is not suited to analyze the dynamic current for devices in a transient state, because the shape of the curve is often complicated owing to the coexistence of the two terms. In order to separate the transient term, we use a voltage waveform with a ramp and plateau region as shown in Fig. 1d. In this scanning mode, first a applied voltage is kept at the start voltage $\left(V_{\text {str }}\right)$. Then, a ramp voltage is applied and both the quasi-static and dynamic currents $\left(C_{\text {eff }} d V / d t+V d C_{\text {eff }} / d t\right)$ are observed. At the hold voltage ( $\left.V_{\text {hold }}\right)$, the ramp is switched to the plateau voltage, and only the $V d C_{\text {eff }} / d t$ term is observed due to $d V / d t=0$. By changing the $V_{\text {hold }}$, we can directly examine the carrier properties in various states of the device.

\section{Experiment}

The structure of the device under study is $\mathrm{Au}(50 \mathrm{~nm}) / \mathrm{Pn}(100 \mathrm{~nm}) / \mathrm{SiO}_{2}$ $(100 \mathrm{~nm}) / \mathrm{p}$-type Si (resistivity $\sim 0.02 \Omega \cdot \mathrm{cm}$ ). The organic layer and the $\mathrm{Au}$ electrode were successively fabricated using a conventional vacuum evaporation technique on a highly doped p-type Si substrate covered with $\mathrm{SiO}_{2}$. The evaporation rate of the organic and metal layers was about $0.5 \AA / \mathrm{s}$. The devices were transferred to a vacuum probe station and DCM was performed in the dark at room temperature. During the transfer of the device from the fabrication chamber to the vacuum probe station, the devices were exposed to air for a short time. We fabricated two kinds of devices, denoted as devices $\mathrm{A}$ and $\mathrm{B}$. The electrode area of device $\mathrm{A}$ is the same as the Pn area $\left(2.4 \mathrm{~mm}^{2}\right)$, while that of device $\mathrm{B}\left(1.77 \mathrm{~mm}^{2}\right)$ is smaller than the Pn area 
$\left(4.91 \mathrm{~mm}^{2}\right)$. Table 1 shows the area of $\mathrm{Au}\left(S_{\mathrm{Au}}\right)$ and $\mathrm{Pn}\left(S_{\mathrm{Pn}}\right)$, and the effective capacitances estimated from the device structure (dielectric constant of $\varepsilon_{\mathrm{Pn}}=4.0$ [22] and $\varepsilon_{\mathrm{SiO}_{2}}=3.8$ ). $C_{\text {dep }}$ shows the geometrical capacitance of the device in depletion, normalized by the electrode area, and corresponds to the capacitance of Region (a) in Fig. 2. $C_{\text {inj }}$ shows the capacitance of the device with the carrier injection from the Au electrode to the Pn layer and corresponds to the capacitance of Region (b) in Fig. 2. $C_{\text {acc }}$ shows the capacitance of the device in which carriers accumulate over the entire $\mathrm{Pn} / \mathrm{SiO}_{2}$ interface and corresponds to the capacitance of Region (d) in Fig. 2.

DCM measurements were performed using the FCE-1 measurement system for ferroelectrics (Toyo Corp.) at Chiba University. The DCM curves were measured by applying biases within $\pm 20 \mathrm{~V}$ to the p-type Si layer, with reference to the $\mathrm{Au}$ electrode. To investigate the frequency dependence of the DCM curve, the ramp rate of a triangular wave voltage was changed from $80 \mathrm{~V} / \mathrm{s}$ to $800 \mathrm{kV} / \mathrm{s}$. Impedance measurements were performed using a frequency response analyzer (Solartron SI 1260 Impedance/Gain-Phase Analyzer) combined with a dielectric interface (Solartron 1296) at Augsburg University. The amplitude of the $\mathrm{AC}$ bias was set to $0.1 \mathrm{~V}$ for the $C$ - $f$ measurement and the DC bias was varied from -20 to $20 \mathrm{~V}$.

Table 1: Area and capacitance of devices A and B. The units of area and capacitance are $\mathrm{mm}^{2}$ and $\mathrm{mF} / \mathrm{m}^{2}$, respectively.

\begin{tabular}{|c|c|c|c|c|c|}
\hline & $S_{\mathrm{Au}}$ & $S_{\mathrm{Pn}}$ & $C_{\text {dep }}$ & $C_{\text {inj }}$ & $C_{\text {acc }}$ \\
\hline \hline Device A & 2.4 & 2.4 & 0.17 & 0.34 & - \\
\hline Device B & 1.77 & 4.91 & 0.17 & 0.34 & 0.93 \\
\hline
\end{tabular}

\section{Results and discussion}

\subsection{Analysis of DCM curves in conventional mode}

Figure 2 shows the typical DCM curves of devices A and B at a ramp rate of $80 \mathrm{~V} / \mathrm{s}$. The inset shows the comparison of the results between DCM and $C-V$ measurement. Vertical axis is the current which is normalized by the electrode area divided by the ramp rate, corresponding to $C_{\text {eff }}$ of a device with unit area. As shown in the inset (Fig. 2), the peak structure can only 
be observed for DCM. This is a unique feature of DCM and we will discuss its results in detail below.

In device $\mathrm{A}$, the capacitance is equal to $C_{\text {dep }}$ at voltages larger than $2 \mathrm{~V}$, indicating that the device is depleted. In the backward scan, the negative current increases at $2 \mathrm{~V}$ owing to the hole injection from the Au electrode to the Pn layer. $C_{\text {eff }}$ at voltages below $-1 \mathrm{~V}$ is consistent with $C_{\text {inj }}$ [Table 1]. Then, in the forward scan, the injected carriers are discharged from the Pn to the Au electrode. Finally, the Pn layer is fully depleted and back to its initial state. In device A, the shape of the DCM curve in the forward scan is almost the same as that in the backward scan.

At voltages larger than $12 \mathrm{~V}$ in the backward scan, the DCM curve of device $\mathrm{B}$ is precisely equal to that of device $\mathrm{A}$. When scanning the voltage to the negative side, the increase of the negative current owing to the hole injection appears in Region (b). The voltage of hole injection for device $\mathrm{B}$ is different from that for device $\mathrm{A}$. This is maybe due to the effect of oxygen on Pn layer: since the Pn layer of device B is not fully covered by the top electrode, the hole injection property may be affected by the oxygen penetrated into the device. Finally, a larger capacitance about $0.8 \mathrm{mF} / \mathrm{m}^{2}$ is observed in Region (d) after the current peak [Fig. 2 Region (c)]. The capacitance estimated from the DCM curves indicates that the charge sheet is formed over the $\mathrm{Pn} / \mathrm{SiO}_{2}$ interface. After the sweep direction of the applied voltage is changed to forward at $-20 \mathrm{~V}$, the current gradually decreases owing to hole discharge [Fig. 2 Region (e)]. The current continues to decay even in the positive bias region in device $\mathrm{B}$, while the carrier discharges around the zero bias region in device $\mathrm{A}$.

A peak structure is observed in Region (c), and it appears only in the DCM curve of device B. In device A, since the area of the Pn layer is the same as that of the top electrode, no carrier spreading along the $\mathrm{Pn} / \mathrm{SiO}_{2}$ interface can be expected after the carrier injection. Thus, we conclude that the current peak originates from the carrier-spreading effect at the $\mathrm{Pn} / \mathrm{SiO}_{2}$ interface. In addition, the slow discharge process in device $\mathrm{B}$ is also owing to its device structure because it is expected that the holes injected farther from the electrode will take longer to discharge.

Figure 3 shows the DCM curves as a function of the frequency of the applied voltage in device A (left) and device B (right). The ramp rate of the triangular wave voltage is changed from $80 \mathrm{~V} / \mathrm{s}$ to $800 \mathrm{kV} / \mathrm{s}$. In device A, although there is no difference in the DCM curves from $80 \mathrm{~V} / \mathrm{s}$ to $8 \mathrm{kV} / \mathrm{s}$, the hole injection voltage shifts slightly to the negative voltage side at 80 
and $800 \mathrm{kV} / \mathrm{s}$ with the increasing frequency [Fig. 3a]. On the other hand, the peak structure in device $\mathrm{B}$ strongly depends on the ramp rate and finally disappears at $800 \mathrm{kV} / \mathrm{s}$ [Fig. 3c]. This result implies that the relaxation time of the carrier-spreading process at the $\mathrm{Pn} / \mathrm{SiO}_{2}$ interface is longer than that of the carrier injection process from the electrode to the Pn layer.

In device $\mathrm{B}$, we can observe the terrace structure, $-0.35 \mathrm{mF} / \mathrm{m}^{2}$, corresponding to the $C_{\text {inj }}$ at $80 \mathrm{~V} / \mathrm{s}$ in the backward scan, that is, the injected holes first accumulate only under the electrode area and then start to spread laterally along the $\mathrm{Pn} / \mathrm{SiO}_{2}$ interface. This result indicates that the injected holes do not spread smoothly along the $\mathrm{Pn} / \mathrm{SiO}_{2}$ interface; this behavior can be explained as follows. When the voltage is applied to the sample, the electric field far from the electrode is not enough to drive the carriers laterally. Therefore, the potential difference between just under and far from the electrode prevents the injected holes from spreading at the $\mathrm{Pn} / \mathrm{SiO}_{2}$ interface. With increasing carrier density, the diffusion effect helps the holes to spread.

At the beginning of the forward scan in device $\mathrm{A}$, the injected holes are discharged smoothly at a lower ramp rate than those discharged at $8 \mathrm{kV} / \mathrm{s}$. In comparison, the shape of the DCM curves does not rise sharply at the apex of the triangular voltage $(-20 \mathrm{~V})$ for higher ramp rates, and the start voltage of the discharge shifts to the positive voltage side in Fig. 3b. This characteristic feature is also observed in device $\mathrm{B}$ at $800 \mathrm{kV} / \mathrm{s}$. In addition, the rounded structure is observed only for device $\mathrm{B}$ when the holes which are located at the $\mathrm{Pn} / \mathrm{SiO}_{2}$ interface far from the electrode are discharged in the forward scan. These results indicate that the injected holes cannot follow the high frequency of the applied voltage.

\subsection{Transient current characteristics during carrier spreading}

Next, we discuss the results of the combined waveform DCM method. Figures $4 \mathrm{a}$ and $\mathrm{b}$ show the combined waveform DCM curves for devices A and $\mathrm{B}$, respectively. Vertical axis is the logarithm of the absolute value of current normalized by the electrode area. The measurement was performed while changing the hold voltage $\left(V_{\text {hold }}\right)$ from 8 to $-10 \mathrm{~V}$ in device $\mathrm{A}$ and from 14 to $-18 \mathrm{~V}$ in device $\mathrm{B}$, and the $V_{\text {str }}$ of device $\mathrm{A}$ and $\mathrm{B}$ is set to 10 and $20 \mathrm{~V}$, respectively. The $V_{\text {hold }}$ is indicated by the bold number in Figs. $4 \mathrm{a}$ and $\mathrm{b}$. The current density in the ramp region (before the voltage is held) corresponds to that measured by the traditional scanning mode in the backward scan. 
In device A, while the $V_{\text {hold }}$ is higher than $2 \mathrm{~V}$, the Pn layer is completely depleted, as shown in Fig. 4a. At a $V_{\text {hold }}$ lower than $0 \mathrm{~V}$, the holes are injected from the electrode and accumulate at the $\mathrm{Pn} / \mathrm{SiO}_{2}$ interface. Just after the voltage is held, the current with various $V_{\text {hold }}$ values decays immediately. If we define that $t_{\text {rel }}$ is the time required for the current to decay less than 0.1 $\mathrm{mA} / \mathrm{m}^{2}$ from the beginning of the plateau voltage region, $t_{\mathrm{rel}}$ is less than 0.1 sec in device $\mathrm{A}$.

In Fig. 4b, the Pn layer is fully depleted around $14 \mathrm{~V}$ (depletion). While decreasing the $V_{\text {hold }}$, the current increases with a hole injection at $10 \mathrm{~V}$ (injection), and then, at $V_{\text {hold }}=2 \mathrm{~V}$, the injected holes start to spread laterally along the $\mathrm{Pn} / \mathrm{SiO}_{2}$ interface. Finally, the charge sheet is completed over the interface at a $V_{\text {hold }}$ lower than $-10 \mathrm{~V}$ (accumulation). Note that the transient currents in the quasi-static, for example, depletion, injection, and accumulation states, decay immediately just after the voltage is held. On the other hand, it takes about 1 sec for the transient current to decay at $V_{\text {hold }}=2$ and $-2 \mathrm{~V}$ in device $\mathrm{B}$. This $t_{\text {rel }}$ is much longer than that in the quasi-static state.

The above result demonstrates that we succeeded in extracting the dynamic current by using the combined waveform DCM method. The dynamic current with a large $t_{\text {rel }}$ continues to flow during the hole-spreading at the $\mathrm{Pn} / \mathrm{SiO}_{2}$ interface, clearly indicating that the peak structure originates from the dynamic current owing to the $V d C / d t$ term. After the charge sheet is formed completely at the $\mathrm{Pn} / \mathrm{SiO}_{2}$ interface $(V<-10), t_{\text {rel }}$ becomes much smaller. In this way, DCM is able to evaluate both quasi-static and dynamic carrier behavior in organic devices.

Figures $4 \mathrm{c}$ and d show the capacitance-frequency $(C-f)$ curves of devices $\mathrm{A}$ and $\mathrm{B}$, respectively. The $\mathrm{DC}$ bias is changed from -20 to $20 \mathrm{~V}$ and is indicated by bold numbers. For frequencies higher than $10^{3} \mathrm{~Hz}$, the capacitance of device A almost agrees with the $C_{\text {dep }}$, because the injected holes cannot follow the frequency of the applied voltage. As the frequency slows, if the DC bias is larger than the hole injection voltage, the capacitance of the device increases and corresponds to $C_{\mathrm{inj}}$. The frequency at the inflection point is called the relaxation frequency $\left(f_{\text {rel }}\right)$. The $f_{\text {rel }}$ at DC bias $=-10$ and $-5 \mathrm{~V}$ is $129 \mathrm{~Hz}$. In device $\mathrm{A}$, one $f_{\text {rel }}$ is observed between the depletion and injection states and reflects the hole injection speed from the electrode to the Pn layer.

In device $\mathrm{B}$, the Pn layer is also depleted at frequencies higher than $10^{3}$ $\mathrm{Hz}$, as in device A. For lower frequencies, the capacitance starts to increase 
from a few hundred hertz; for example, $f_{\text {rel }}=167 \mathrm{~Hz}$ at $\mathrm{DC}$ bias $=-5$ and $0 \mathrm{~V}$. This $f_{\text {rel }}$ is comparable to that estimated for device $\mathrm{A}$; this result indicates that the holes accumulated just under the electrode are following the frequency of the applied voltage. When lowering the frequency, a second increase of the capacitance can be observed at DC bias $=0 \mathrm{~V}$. Since the capacitance almost corresponds to $C_{\text {acc }}$ at the low frequency region, this second $f_{\text {rel }}$ reflects the hole spreading speed along the $\mathrm{Pn} / \mathrm{SiO}_{2}$ interface. The inverse of the relaxation frequency is about $1 \mathrm{sec}$, by inverting the observed $f_{\text {rel }}$ of $1 \mathrm{~Hz}$. This value corresponds well with $t_{\text {rel }}$ of combined waveform DCM. This result demonstrates that the combined waveform DCM method is a useful tool to observe not only the effective capacitance of a device but also the relaxation time, as impedance spectroscopy.

\section{Conclusion}

In the DCM of a Pn MIS structure, the carrier injection from the electrode to the organic layer spreading along the $\mathrm{Pn} / \mathrm{SiO}_{2}$ interface and the accumulation at the interface were clearly observed. The combined waveform DCM method was proposed and the quasi-static $(C d V / d t)$ and dynamic $(V d C / d t)$ currents of DCM were successfully separated in the plateau voltage region. We demonstrated that a current peak observed in the DCM curve originates from the carrier spreading along the $\mathrm{Pn} / \mathrm{SiO}_{2}$ interface. The transient current with a very long decay time was found during the formation of the charge sheet at the $\mathrm{Pn} / \mathrm{SiO}_{2}$ interface. Its decay time is consistent with $t_{\text {rel }}$ estimated from the $C-f$ measurement. These results indicate that DCM is a powerful tool for investigating the quasi-static and dynamic behavior of carriers in organic devices. By using various types of combined waveforms, we can expect further extensions of DCM to investigate carrier behavior in detail.

\section{ACKNOWLEDGMENTS}

We thank Dr. S. Ogawa for his helpful suggestions. This work was supported by the Global-COE program at Chiba University (Advanced School for Organic Electronics, G-03, MEXT), KAKENHI (Grants No. 21245042, 22750167), and the Funding Program for World-Leading Innovative R\&D on Science and Technology (FIRST). YT gratefully acknowledges JSPS Research Fellowships for Young Scientists (Grants No. 22.7042). WB also acknowledges financial support by JSPS. 


\section{References}

[1] G. Horowitz, Adv. Mater. 10 (1998) 365-377.

[2] C.D. Dimitrakopoulos, P.R.L. Malenfant, Adv. Mater. 14 (2002) 99-117.

[3] S.A. DiBenedetto, A. Facchetti, M.A. Ratner, T.J. Marks, Adv. Mater. 21 (2009) 1407-1433.

[4] S. Ogawa, Y. Kimura, H. Ishii, M. Niwano, Jpn. J. Appl. Phys. 42 (2003) L1275-L1278.

[5] S. Ogawa, T. Naijo, Y. Kimura, H. Ishii, M. Niwano, Synth. Met. 153 (2005) 253-256.

[6] Y. Suzue, T. Manaka, M. Iwamoto, Jpn. J. Appl. Phys. 44 (2005) 561565.

[7] T. Manaka, E. Lim, R. Tamura, M. Iwamoto, Thin Solid Films 499 (2006) 386-391.

[8] S.M. Sze, Physics of Semiconductor Devices, second ed., John Wiley \& Sons, New York, 1981.

[9] S. Scheinert, W. Schliefke, Synth. Met. 139 (2003) 501-509.

[10] Y.-M. Chen, C.-F. Lin, J.-H. Lee, J. Huang, Solid-State Electron. 52 (2008) 269-274.

[11] T. Manaka, C.-Q. Li, X.-M. Cheng, M. Iwamoto, J. Chem. Phys. 120 (2004) 7725-7732.

[12] T. Manaka, E. Lim, R. Tamura, D. Yamada, M. Iwamoto, Appl. Phys. Lett. 89 (2006) 072113.

[13] T. Manaka, E. Lim, R. Tamura, M. Iwamoto, Nat. Photon. 1 (2007) 581-584.

[14] A.J. Twarowski, A.C. Albrecht, J. Chem. Phys. 70 (1979) 2255-2261.

[15] K. Iriyama, M. Shiraki, K. Thuda, A. Okada, M. Sugi, S. Iizima, K. Kudo, S. Shiokawa, T. Moriizumi, T. Yasuda, Proc. 1st Photovoltaic Science and Engineering Conf. Japan, Tokyo, 1979, Jpn. J. Appl. Phys. 19 (1980) Suppl. 19-2, p. 173. 
[16] S. Egusa, N. Gemma, M. Azuma, J. Appl. Phys. 71 (1992) 2042-2044.

[17] S. Egusa, A. Miura, N. Gemma, M. Azuma, Jpn. J. Appl. Phys. 33 (1994) 2741-2745.

[18] Y. Noguchi, N. Sato, Y. Tanaka, Y. Nakayama, H. Ishii, Appl. Phys. Lett. 92 (2008) 203306.

[19] Y. Noguchi, N. Sato, Y. Miyazaki, H. Ishii, Appl. Phys. Lett. 96 (2010) 143305.

[20] S. Ogawa, T. Naijo, Y. Kimura, H. Ishii, M. Niwano, Appl. Phys. Lett. 86 (2005) 252104.

[21] Y. Liang, C.D. Frisbie, H.-C. Chang, P.P. Ruden, J. Appl. Phys. 105 (2009) 024514.

[22] A. Bolognesi, M. Berliocchi, M. Manenti, A. D. Carlo, P. Lugli, K. Lmimouni, C. Dufour, IEEE T. Electron Dev. 51 (2004) 1997-2003. 


\section{Figure captions}

Fig. 1: (a) Schematic illustration of device structure and experimental setup. (b) Top: Applied voltage waveform in conventional DCM. Bottom: Current response with hole injection. (c) A typical C-V curve for a p-type semiconductor with hole injection. (d) Top: Applied voltage waveform in combined waveform DCM. Bottom: Current response with hole injection and transient current.

Fig. 2: Device structure dependence in DCM curves of an MIS diode (device A: dashed line, device B: solid line). Region (a): Without carrier injection. Region (b): Carrier injection from Au electrode to Pn layer. Region (c): Injected carrier spreading along the $\mathrm{Pn} / \mathrm{SiO}_{2}$ interface. Region (d): Carrier accumulation over the interface. Region (e): Carrier discharge from Pn layer to $\mathrm{Au}$ electrode. Inset: Comparison of DCM (solid line) with $C$ - $V$ curves (dashed line) for device B.

Fig. 3: DCM curves as a function of frequency of applied voltage in device A (left) and device B (right). Ramp rate is varied from $80 \mathrm{~V} / \mathrm{s}$ to 800 $\mathrm{kV} / \mathrm{s}$. The curves showed characteristic changes with increasing frequency: (a) Shift of the hole injection voltage. (b) Shift of the start voltage of hole discharge. (c) Shift of the peak structure. (d) Rounded structure with hole discharge.

Fig. 4: Combined waveform DCM curves of device A (a) and device B (b) with the various $V_{\text {hold }}$. Ramp rate is $8 \mathrm{~V} / \mathrm{s}$ and bold numbers show the $V_{\text {hold }}$. $C$ - $f$ curves of device A (c) and device B (d). Bold numbers show the $\mathrm{DC}$ bias and $f_{\text {rel }}$ shows the relaxation frequency. 
(a)

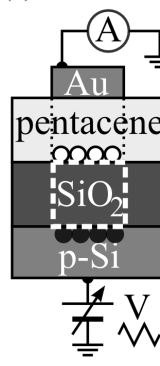

(b)

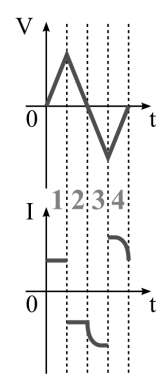

(c)

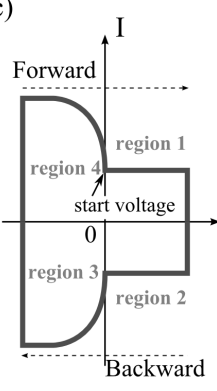

(d)

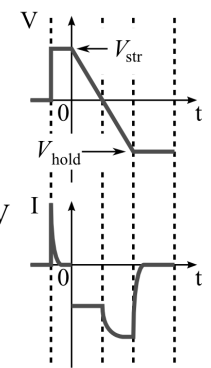

Figure 1: (a) Schematic illustration of device structure and experimental setup. (b) Top: Applied voltage waveform in conventional DCM. Bottom: Current response with hole injection. (c) A typical C-V curve for a p-type semiconductor with hole injection. (d) Top: Applied voltage waveform in combined waveform DCM. Bottom: Current response with hole injection and transient current.

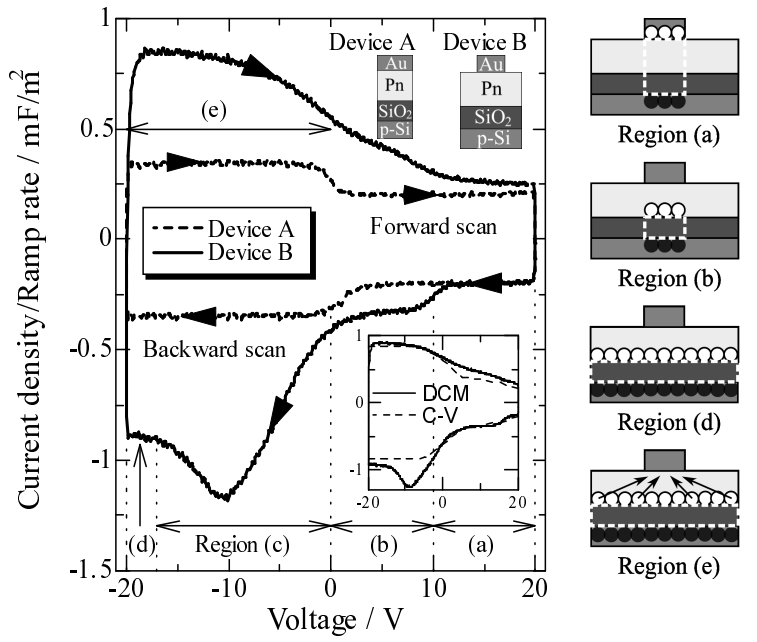

Figure 2: Device structure dependence in DCM curves of an MIS diode (device A: dashed line, device B: solid line). Region (a): Without carrier injection. Region (b): Carrier injection from $\mathrm{Au}$ electrode to Pn layer. Region (c): Injected carrier spreading along the $\mathrm{Pn} / \mathrm{SiO}_{2}$ interface. Region (d): Carrier accumulation over the interface. Region (e): Carrier discharge from Pn layer to Au electrode. Inset: Comparison of DCM (solid line) with $C$ - $V$ curves (dashed line) for device B. 

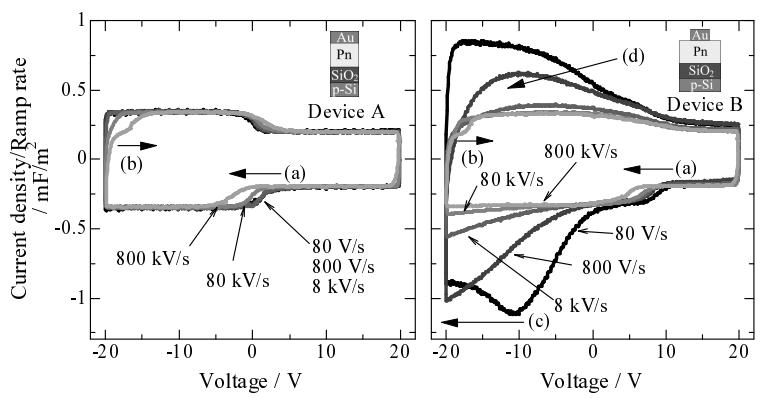

Figure 3: DCM curves as a function of frequency of applied voltage in device A (left) and device B (right). Ramp rate is varied from $80 \mathrm{~V} / \mathrm{s}$ to $800 \mathrm{kV} / \mathrm{s}$. The curves showed characteristic changes with increasing frequency: (a) Shift of the hole injection voltage. (b) Shift of the start voltage of hole discharge. (c) Shift of the peak structure. (d) Rounded structure with hole discharge.
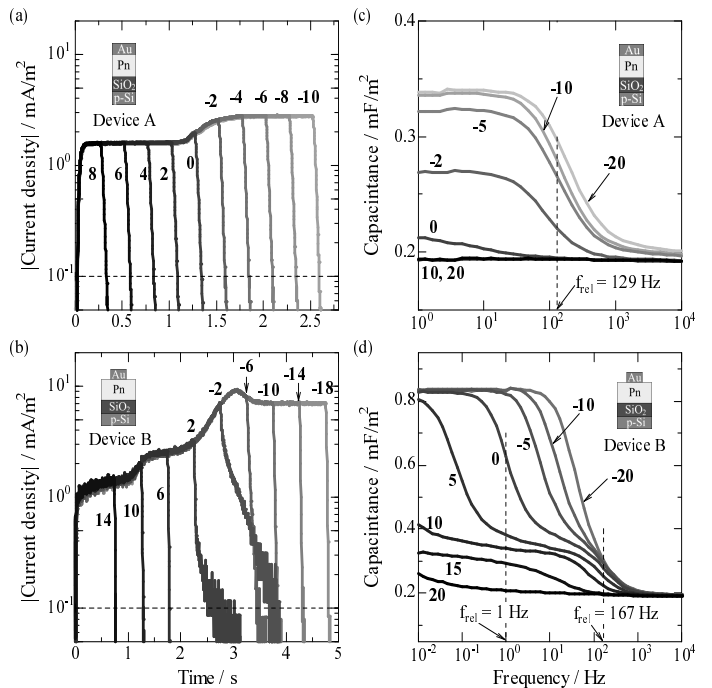

Figure 4: Combined waveform DCM curves of device A (a) and device B (b) with the various $V_{\text {hold }}$. Ramp rate is $8 \mathrm{~V} / \mathrm{s}$ and bold numbers show the $V_{\text {hold }}$. $C$-f $f$ curves of device A (c) and device B (d). Bold numbers show the DC bias and $f_{\text {rel }}$ shows the relaxation frequency. 